\title{
TOPOLOGY OF INJECTIVE ENDOMORPHISMS OF REAL ALGEBRAIC SETS
}

\author{
ADAM PARUSIŃSKI
}

\begin{abstract}
Using only basic topological properties of real algebraic sets and regular morphisms we show that any injective regular self-mapping of a real algebraic set is surjective. Then we show that injective morphisms between germs of real algebraic sets define a partial order on the equivalence classes of these germs divided by continuous semi-algebraic homeomorphisms. We use this observation to deduce that any injective regular self-mapping of a real algebraic set is a homeomorphism. We show also a similar local property. All our results can be extended to arc-symmetric semi-algebraic sets and injective continuous arc-symmetric morphisms, and some results to Euler semi-algebraic sets and injective continuous semi-algebraic morphisms.
\end{abstract}

In 1960 Newman [16] showed that any injective real polynomial map $\mathbb{R}^{2} \rightarrow \mathbb{R}^{2}$ is surjective. This was extended to the real polynomial maps $\mathbb{R}^{n} \rightarrow \mathbb{R}^{n}$ in 1962 by Białynicki-Birula and Rosenlicht [5]. In 1969 Ax [3] showed that any injective regular self-mapping of a complex algebraic variety is surjective. Unilike the proof of [5], that was topological, the proof of $\mathrm{Ax}$ is based on the Lefschetz principle and a reduction to the finite field case. In [7] (1969) Borel extended the idea of [5] and gave a topological proof of Ax' Theorem that can be applied to real algebraic non-singular varieties. The first proof of an analogous result for singular real algebraic varieties was given in 1999 by Kurdyka 11. Kurdyka's proof is based on Borel's argument and the geometry of semi-algebraic arc-symmetric sets. For more on history of the problem of surjectivity of injective mappings, the motivation, and a wide spectrum of possible applications we refer the reader to a recent paper of Gromov [9]. We note that Borel's proof gives as well that an injective regular self-mapping of a complex algebraic variety or of a non-singular real algebraic variety is a homeomorphism. The analogous statement in the general real algebraic case has not been proven till now (to the author's best knowledge) and doesn't not follow from [11].

In this paper we first present a new topological proof of Kurdyka's theorem and then extend the argument to show that injective regular self-mappings of real algebraic varieties are homeomorphisms. Moreover we obtain local versions of this result and establish a hierarchy of real algebraic singularities (more generally of Euler semi-algebraic singularities) with respect to injective regular mappings.

Our approach is based on two classical topological properties of real algebraic sets and maps. The first one is Sullivan's Theorem that each real algebraic set is Euler (see definition 1.1 below). The second one says that for a regular map $f: X \rightarrow Y$ of real algebraic sets there exists a proper real algebraic subset $Y^{\prime} \subset Y$ such that the Euler characteristic of the fibres of $f$, taken modulo 2, is constant on $Y \backslash Y^{\prime}$. This is the crucial observation for the problem since it implies that the image of injective regular map of

1991 Mathematics Subject Classification. 14Pxx, 14A10, 32B10. 
real algebraic varieties is algebraically constructible, i. e. belongs to the Boolean algebra generated by real algebraic sets.

In section 11 we recall basic properties of Euler semi-algebraic sets. In particular, these sets have a well-defined fundamental class in the Borel-Moore homology with $\mathbb{Z}_{2}$ coefficients. As an easy corollary, proposition 1.3, we obtain that any injective continuous semi-algebraic self-map of a compact Euler semi-algebraic set is a homeomophism and an analogous local version. The class of algebraically constructible sets is introduced in section 2. Its topological properties, similar to the ones of real algebraic sets are established in sections 2 and 4 . The sujectivity theorem of Kurdyka is proven in section 3. The proof is based on Borel's argument in non-singular case and the hunt for invariant subsets. More precisely, let $X$ be a real algebraic set and let $f: X \rightarrow X$ be injective regular. We show that there is a subset $\tilde{\Sigma} \subset X$, of dimension smaller than $X$, such that $f(\tilde{\Sigma}) \subset \tilde{\Sigma}$ and $U=X \backslash \tilde{\Sigma}$ is a topological manifold. We do not know whether such $\tilde{\Sigma}$ can be found algebraic, but the set we construct is algebraically constructible and closed in $X$. Then we apply the induction on dimension to $\left.f\right|_{\tilde{\Sigma}}: \tilde{\Sigma} \rightarrow \tilde{\Sigma}$ to show that it is surjective (this step shows that we have to extend the category we work with from the algebraic sets to the algebraically constructible ones). Then $f(U) \subset U$ and we conclude that $f(U)=U$ by Borel's argument.

In section 5 we study germs of real algebraic sets (or more generally of Euler semialgebraic sets) and injective mappings between them. For two such germs we write $(Y, y) \prec$ $(X, x)$ if there is a germ $f:(Y, y) \rightarrow(X, x), f$ being continuous, injective, and semialgebraic. We show that $\prec$ is a partial order (on the set of germs up to semi-algebraic homeomorphisms). One may think that $(Y, y) \prec(X, x)$ means that the singularity type of $(Y, y)$ is less complicated than that of $(X, x)$. There are two precise result that justify this interpretation. Firstly a non-singular germ is minimal (but not necessarily unique as such) among the germs of a fixed dimension, corollary 1.7. Secondly, if $x \in X$ then the singularity type of $\left(X, x^{\prime}\right)$ for $x^{\prime}$ close to $x$ cannot be more complicated than that of $(X, x)$, i. e. if $(X, x) \prec\left(X, x^{\prime}\right)$ then both germs are homeomorphic. Using this hierarchy of singularities we show the main result that any injective (and so bijective) regular self-map of real algebraic set is a homeomorphism, theorem 5.7. (Surprisingly, in the case of sets of pure-dimension, the assumption can be weaken considerably: any bijective continuous semi-algebraic self-map of a pure-dimensional Euler semi-algebraic set is a homeomorphism, cf. theorem 5.6.)

In the paper we formalize what properties of algebraically constructible sets we need by introducing the notion of constructible category of semi-algebraic sets, definition 2.3, and then we proceed in any such category. Though this makes some proofs, e. g. of theorem 2.7, more involved it has the advantange of extending automatically the results of the paper to semi-algebraic arc-symmetric sets, cf. subsection 4.2 . It gives also a bridge to the approach of Kurdyka, though we use only basic topological properties of arcsymmetric sets. Algebraically constructible sets form the smallest constructible category, arc-symmetric sets the biggest one, see section 4.3. In section 1 we relate these categories to the integration with respect to the Euler characteristic and the algebraically constructible and the Nash-constructible functions.

Notation and terminology. In this paper a "regular set" means a pure-dimensional topological manifold and a "stratification" means a finite decomposition into such regular, not necessarily connected, sets. The Zariski closure of $X$ will be denoted by $\bar{X}^{Z}$. 


\section{TOPOLOGICAL PRELIMINARIES}

By a semi-algebraic set we mean a semi-algebraic subset of an affine space $\mathbb{R}^{N}$, or of a projective space $\mathbb{R P}^{N}$. We often, but not always, assume that a semi-algebraic set is locally compact, that is locally closed as a subset of $\mathbb{R}^{N}$ or $\mathbb{R P}^{N}$. A semi-algebraic map is a map between two semi-algebraic sets with semi-algebraic graph. Semi-algebraic maps may be continuous or not. Similarly, by algebraic sets we mean the algebraic subsets of affine or projective spaces. Our discussion can be extended to semi-algebraic and algebraic subsets of real algebraic varieties that we will not do for simplicity of exposition. For the background on semi-algebraic and real algebraic sets we refer the reader to 㽢.

We say that a semi-algebraic set $X$ is (topologically) regular of dimension $d$ at $x \in X$ if there is a neighbourhood of $X$ in $x$ that is a topological manifold of dimension $d$. In this paper we shall drop the word "topologically" and call topologically regular sets regular. We say that $X$ is regular at $x$ if it is regular of dimension $d=\operatorname{dim} X$. In this case we also say that $x$ is a regular point of $X . X$ is regular if it is regular at all its points.

In this section we discuss basic topological properties of Euler semi-algebraic sets. Let $X$ be semi-algebraic and $x \in X$. The local Euler-Poincaré characteristic of $X$ at $x$ is, by definition,

$$
\chi(X, X \backslash x)=\sum_{i}(-1)^{i} \operatorname{dim} H_{i}\left(X, X \backslash x ; \mathbb{Z}_{2}\right) .
$$

Definition 1.1. We call a locally compact semi-algebraic set $X$, Euler if $\chi(X, X \backslash x)$ is odd for all $x \in X$.

Let $X \subset \mathbb{R}^{N}$ and let $S(x, \varepsilon)$ be the sphere of radius $\varepsilon>0$ centered at $x$. By the local conic structure lemma, cf. [4] (9.3.6), for $\varepsilon$ sufficiently small the topological type of $S(x, \varepsilon) \cap X$ is independent of $\varepsilon$. This space is called the link of $x$ in $X$. It is easy to check that $\chi(X, X \backslash x)$ is odd iff the Euler characteristic of the link of $X$ at $x$ is even. By Sullivan's theorem [19] each real algebraic set is Euler.

Let $X$ be a locally compact semi-algebraic set. Consider the Borel-Moore homology $H^{B M}(X)$ of $X$, see [8]. Since $X$ can be triangulated the Borel-Moore homology of $X$ is isomorphic to the simplicial homology of $X$ with closed supports (i.e. using possibly infinite simplicial chains). If $X^{\prime}$ is a compact space containing $X$ such that $X^{\prime}$ is triangulated with $X^{\prime} \backslash X$ as a subcomplex, then the Borel-Moore homology of $X$ is isomorphic to the relative simplicial homology of the pair $\left(X^{\prime}, X^{\prime} \backslash X\right)$. In particular if $\bar{X}=X \cup\{\infty\}$ is the one point compactification of $X$, that can be identified with a semi-algebraic set, cf. [4] (2.5.9), we have

$$
H_{i}^{B M}\left(X ; \mathbb{Z}_{2}\right)=H_{i}\left(\bar{X},\{\infty\} ; \mathbb{Z}_{2}\right) .
$$

For an open semi-algebraic $U \subset X$ there is a restriction homomorphism

$$
H_{i}^{B M}\left(X ; \mathbb{Z}_{2}\right) \rightarrow H_{i}^{B M}\left(U ; \mathbb{Z}_{2}\right)
$$

Then $Y=X \backslash U$ is a closed semi-algebraic subset of $X$ and the exact sequence of a triple for classical homology gives an exact sequence, cf. [4] (11.7.12),

$$
\cdots \rightarrow H_{i+1}^{B M}\left(U ; \mathbb{Z}_{2}\right) \rightarrow H_{i}^{B M}\left(Y ; \mathbb{Z}_{2}\right) \rightarrow H_{i}^{B M}\left(X ; \mathbb{Z}_{2}\right) \rightarrow H_{i}^{B M}\left(U ; \mathbb{Z}_{2}\right) \rightarrow \cdots
$$

Semi-algebraic sets can be triangulated, a compact semi-algebraic set $X$ is homeomorphic to a finite simplicial complex. Let $\operatorname{dim} X=n$. If $X$ is Euler then for each $(n-1)$-dimensional simplex $\tau$ there is an even number of $n$-dimensional simplices that 
contain $\tau$ (we shall consider all simplices closed). This shows that the formal sum of $n$ simplices forms a $\mathbb{Z}_{2}$-cycle. Its class in $\mathrm{H}_{n}\left(X ; \mathbb{Z}_{2}\right)$ is non-zero. It is called the fundamental class of $X$ and denoted by $[X]$. It is characterized by the property that its image under $\mathrm{H}_{n}\left(X ; \mathbb{Z}_{2}\right) \rightarrow \mathrm{H}_{n}\left(X, X \backslash x ; \mathbb{Z}_{2}\right)$ is nonzero for all $x$ regular in $X$. The fundamental class is independent of the choice of triangulation.

If $X$ is locally compact and Euler then $\bar{X}$ is Euler. This is a simple exercise. We provide a proof for the reader convenience.

Proposition 1.2. The one point compactification of an Euler semi-algebraic set is Euler.

Proof. Triangulate $\bar{X}$ so that $\{\infty\}$ is a simplex. A triangulated space is Euler if for each simplex $\sigma$ the number of simplices $\tau$ such that $\sigma \subset \tau, \sigma \neq \tau$, is even. Note that each k-simplex $\tau$ contains exactly $2^{k+1}-2$ non-empty simplices $\sigma$ such that $\sigma \neq \tau$. Thus, if we count the number of relations $\sigma \subset \tau, \sigma \neq \tau$, among non-empty simplices, we get an even number. Hence there is always an even number of simplices $\sigma$ having an odd number of adherent simplices $\tau$, i. e. $\sigma \subset \tau, \sigma \neq \tau$. This shows the proposition since for the given triangulation of $\bar{X}$ all simplices except maybe $\{\infty\}$ have an even number of adherent simplices by assumption.

For a locally compact semi-algebraic set of dimension $n$ we define the fundamental class

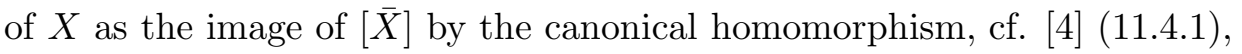

$$
H_{n}\left(\bar{X} ; \mathbb{Z}_{2}\right) \rightarrow H_{n}\left(\bar{X},\{\infty\} ; \mathbb{Z}_{2}\right)=H_{n}^{B M}\left(X ; \mathbb{Z}_{2}\right)
$$

Here are simple general observations related to the problem of surjectivity of injective mappings.

\section{Proposition 1.3.}

(i) Let $f: X \rightarrow X$ be a continuous semi-algebraic self-map of an Euler compact semialgebraic $X$. If $f$ is injective then it is a homeomorphism.

(ii) Let $f:(X, x) \rightarrow(X, x)$ be the germ of a continuous semi-algebraic self-map of an Euler semi-algebraic $X$. Then if $f$ is injective then it is the germ of a local homeomorphism.

Proof. To prove (i) it suffices to show that $f$ is surjective. Suppose that this is not the case. The image $Y=f(X)$ of $f$ is a closed semialgebraic subset of $X$ and $f: X \rightarrow f(X)$ is a homeomorphism. Let $U=X \backslash Y$ and let $d=\operatorname{dim} U$. By (1.2) the following sequence is exact

$$
0 \rightarrow H_{d}^{B M}\left(Y ; \mathbb{Z}_{2}\right) \rightarrow H_{d}^{B M}\left(X ; \mathbb{Z}_{2}\right) \rightarrow H_{d}^{B M}\left(U ; \mathbb{Z}_{2}\right) \rightarrow \cdots
$$

But $X$ and $Y$ are homeomorphic and hence $\operatorname{dim} H_{d}^{B M}\left(X ; \mathbb{Z}_{2}\right)=\operatorname{dim} H_{d}^{B M}\left(X ; \mathbb{Z}_{2}\right)$. This would imply that $H_{d}^{B M}\left(X ; \mathbb{Z}_{2}\right) \rightarrow H_{d}^{B M}\left(U ; \mathbb{Z}_{2}\right)$ is the zero homomorphism that is impossible by the following lemma.

Lemma 1.4. Let $X$ be an Euler locally compact semi-algebraic set and let $U \subset X$ be open semi-algebraic. Then the fundamental class of $U$ is in the image of restriction homomorphism $H_{\operatorname{dim} U}^{B M}\left(X ; \mathbb{Z}_{2}\right) \rightarrow H_{\operatorname{dim} U}^{B M}\left(U ; \mathbb{Z}_{2}\right)$.

Proof. If $\operatorname{dim} U=\operatorname{dim} X$, then it is easy to see that $[U]$ is the image of $[X]$. In the general case let $d=\operatorname{dim} U$. Let $\bar{X}$ be the one point compactification of $X$. Triangulate $\bar{X}$ so that $\bar{X} \backslash U$ is a subcomplex and consider the first barycentric subdivision of $\bar{X}$. Then, by [19], see also [2] proposition 1 , the formal sum of d-simplices is a $\mathbb{Z}_{2}$-cycle. (It represents the $d$-th homology Stiefel-Whitney class of $\bar{X}$.) Its image in $H_{d}\left(X, X \backslash U ; \mathbb{Z}_{2}\right)=H_{d}^{B M}\left(U ; \mathbb{Z}_{2}\right)$ is the fundamental class of $U$. This shows the lemma. 
Let $B(x, \varepsilon)$ be an open ball in $X$ centered at $x$ and of radius $\varepsilon>0$. By the local conic structure, 泪] (9.3.6),

$$
H_{i}^{B M}\left(B(x, \varepsilon) ; \mathbb{Z}_{2}\right) \simeq H_{i}\left(X, X \backslash x ; \mathbb{Z}_{2}\right),
$$

for $\varepsilon>0$ and small. This holds not only for small balls but for any semi-algebraic family of open neighbourhoods of $x$ in $X$ in particular for the familly $f^{-1}(B(x, \varepsilon)), \varepsilon>0$. That is we claim that

$$
H_{i}^{B M}\left(B(x, \varepsilon) ; \mathbb{Z}_{2}\right)=H_{i}^{B M}\left(f^{-1}(B(x, \varepsilon)) ; \mathbb{Z}_{2}\right),
$$

for $\varepsilon>0$ and small. Indeed, this is can be obtain by the following classical argument. Let $\rho: X \rightarrow[0, \infty)$ be given by $\rho(y)=\operatorname{dist}(f(y), x)$. Denote $U(x, \varepsilon)=\rho^{-1}([0, \varepsilon))$. Then, by topological triviality of $\rho$ over $\left(0, \varepsilon_{0}\right)$, for an $\varepsilon_{0}>0, U(x, \varepsilon) \subset U\left(x, \varepsilon^{\prime}\right)$ is a homotopy equivalence if $0<\varepsilon \leq \varepsilon^{\prime} \leq \varepsilon_{0}$. Considering the homomorphism of cohomology groups induced by the inclusions

$$
B\left(x, \varepsilon_{1}\right) \subset U\left(x, \varepsilon_{2}\right) \subset B\left(x, \varepsilon_{3}\right) \subset U\left(x, \varepsilon_{4}\right), \quad 0<\varepsilon_{1} \ll \varepsilon_{2} \ll \varepsilon_{3} \ll \varepsilon_{4} \ll \varepsilon_{0},
$$

we get $H_{i}^{B M}\left(B\left(x, \varepsilon_{1}\right) ; \mathbb{Z}_{2}\right)=H_{i}^{B M}\left(U\left(x, \varepsilon_{2}\right) ; \mathbb{Z}_{2}\right)$ and hence 1.4$)$. (Alternatively one may show $\left.H_{i}^{B M}(U(x, \varepsilon)) ; \mathbb{Z}_{2}\right) \simeq H_{i}\left(X, X \backslash x ; \mathbb{Z}_{2}\right)$ using the local conic structure induced of $X$ at $x$ induced by $\rho$.)

Now (ii) follows from the same argument as (i) applied to $B(x, \varepsilon)$, its closed subset $f(X) \cap B(x, \varepsilon)$, and $U=B(x, \varepsilon) \backslash f(X)$.

Remark 1.5. Note that for the existence of fundamental class it is not necessary to assume tha $X$ is Euler. It suffices that $X$ is Euler in codimension one that is there exists a semi-algebraic subset $Y \subset X, \operatorname{dim} Y \leq \operatorname{dim} X-2$, such that $\chi(X, X \backslash x)$ is odd for every $x \in X \backslash Y$. (This condition is actually equivalent to the existence of fundamental class.) Thus, in particular, proposition 1.3 and lemma 1.4 hold for $X$ Euler in codimension 1 provided it is of pure dimension (then the set $U$ of the proof has the same dimension as $X)$.

We shall need later the following simple observation.

Lemma 1.6. Let $X$ be a connected topological manifold and a semi-algebraic set and let $Y$ be a closed semi-algebraic subset of $X$. If $Y$ is Euler in codimension 1 then either $Y=X$ or $\operatorname{dim} Y<\operatorname{dim} X$.

Proof. Let $n=\operatorname{dim} X$. Suppose $U=X \backslash Y$ is non-empty. Then (1.2) gives an exact sequence

$$
0 \rightarrow H_{n}^{B M}\left(Y ; \mathbb{Z}_{2}\right) \rightarrow H_{n}^{B M}\left(X ; \mathbb{Z}_{2}\right) \rightarrow H_{n}^{B M}\left(U ; \mathbb{Z}_{2}\right) \rightarrow \cdots
$$

By assumption the only non-zero element of $H_{n}^{B M}\left(X ; \mathbb{Z}_{2}\right)$ is $[X]$. Its image in $H_{n}^{B M}\left(U ; \mathbb{Z}_{2}\right)$ equals $[U]$ by lemma 1.4 so is non-zero and hence $H_{n}^{B M}\left(Y ; \mathbb{Z}_{2}\right)=0$. But if $Y$ is Euler in codimension 1 then $H_{\operatorname{dim} Y}^{B M}\left(Y ; \mathbb{Z}_{2}\right) \neq 0$ and therefore $\operatorname{dim} Y<n$.

Corollary 1.7. Let $f:(Y, y) \rightarrow(X, x)$ be an injective continuous and semi-algebraic map, where $(X, x),(Y, y)$ are germs of locally compact semi-algebraic sets, $\operatorname{dim}_{x} X=\operatorname{dim}_{y} Y$. Suppose that $(X, x)$ be a topological manifold and that $(Y, y)$ is Euler in codimension 1. Then $f$ is a local homeomorphism. 


\section{Constructible Categories}

First recall two basic topological properties of real algebraic sets and regular maps.

Theorem 2.1. (Sullivan, [19]) Every real algebraic set is Euler.

Regular maps satisfy the following basic topological mod 2 property (cf. [1] (2.3.2)).

Theorem 2.2. (see for instance [1] (2.3.2)) Let $F: X \rightarrow Y$ be a regular map of real algebraic sets. Then there is a proper algebraic subset $Y^{\prime} \subset Y$ such that the Euler characteristic $\chi\left(F^{-1}(y)\right), y \in Y$, is constant modulo 2 on $Y \backslash Y^{\prime}$.

Definition 2.3. We say that a semi-algebraic set $X$ of $\mathbb{R}^{N}$ or $\mathbb{R P}^{n}$ is algebraically constructible (or Zariski constructible) if $X$ belongs to the Boolean algebra generated by the algebraic subsets of $\mathbb{R}^{N}$. Then we write $X \in \mathcal{A C}$ for short.

The algebraically constructible sets satisfy similar topological properties to the real algebraic sets. For instance it can be easily showed by Sullivan's Theorem that each locally compact algebraically constructible set is Euler. But they form a strictly wider class than the algebraic sets. Closed or even compact algebraically constructible sets are not necessarily algebraic. Moreover the image of a regular injective algebraic morphism is not necessarily algebraic but it is algebraically constructible. Indeed, let $F: X \rightarrow Y$ be a regular injective map of real algebraic sets. Then the Euler characteristic of fibers of $F$ is either 0 or 1. Thus, if $Y^{\prime} \subset Y$ is the subset given by theorem 2.2, then $Y \backslash Y^{\prime}$ is either contained in or disjoint with $F(X)$. Then, by inductive argument on $\operatorname{dim} Y$ and the number of irreducible components of $Y$, applied to the restriction $\left.F\right|_{F^{-1}\left(Y^{\prime}\right)}: F^{-1}\left(Y^{\prime}\right) \rightarrow Y^{\prime}$, we obtain that $F(X)$ is algebraically constructible.

Definition 2.4. Let $\mathcal{C}$ be a sub-collection of semi-algebraic sets. We call a map between two semi-algebraic sets of $\mathcal{C}$ a $\mathcal{C}$-map if its graph is in $\mathcal{C}$. We say that $\mathcal{C}$ is a constructible category if it satisfies the following axioms:

A1. $\mathcal{C}$ contains algebraic sets.

A2. $\mathcal{C}$ is stable by boolean operations $\cap, \cup, \backslash$.

A3. (a) $\mathcal{C}$ is stable by the inverse images by $\mathcal{C}$-maps.

(b) $\mathcal{C}$ is stable by images by injective $\mathcal{C}$-maps.

A4. For each locally compact $X$ in $\mathcal{C}$ there exists a semi-algebraic subset $Y \subset X$, $\operatorname{dim} Y \leq \operatorname{dim} X-2$, such that $X \backslash Y$ is Euler.

Recall after section 1 that the last axioms means that $X$ has a well-defined fundamental class in the Borel-Moore homology with $\mathbb{Z}_{2}$ coefficients.

If a semi-algebraic $X$ belongs to a constructible category $\mathcal{C}$ then we say that $X$ is $\mathcal{C}$ constructible and write $X \in \mathcal{C}$. We shall show in the Section 1 below that the algebraically constructible sets form a constructible category. This is the smallest constructible category. The arc-symmetric sets, see Section 1 below, form the largest constructible category. Using this observation we shall show, see subsection 4.3, that the axioms A1-A4 actually imply that each locally compact set in a constructible category is Euler. Constructible categories, as the algebraically constructible sets or the arc-symmetric sets, share many other interesting properties, as for instance the existence of $\mathcal{C}$-closure.

Theorem 2.5. Let $\mathcal{C}$ be a constructible category. Then the semi-algebraic sets posses a well-defined closure in $\mathcal{C}$. That is for any given locally compact $\mathcal{C}$-constructible set $X$ and 
a semi-algebraic $A \subset X$ there is the smallest closed subset of $X$ that belongs to $\mathcal{C}$ and contains $A$. We denote it by $\bar{A}^{\mathcal{C}}$. Any other closed subset of $X$ that is in $\mathcal{C}$ and contains A must contain $\bar{A}^{\mathcal{C}}$.

Proof. Induction on $\operatorname{dim} X$.

Consider first the case $\operatorname{dim}(X \backslash A)<\operatorname{dim} X$. Replacing $A$ by its topological closure we may suppose $A$ closed in $X$. Let $S=\overline{(X \backslash A)}^{Z}$ denote the Zariski closure of $X \backslash A$. Then $X^{\prime}=S \cap X$ is $\mathcal{C}$-constructible. Set $A^{\prime}=S \cap A$ and define

$$
\bar{A}^{\mathcal{C}}=A \cup{\overline{A^{\prime}}}^{\mathcal{C}}=(X \backslash S) \cup{\overline{A^{\prime}}}^{\mathcal{C}}
$$

where $\overline{A^{\prime}}$ is the $\mathcal{C}$-closure of $A^{\prime}$ in $X^{\prime}$ that exists by the inductive hypothesis. The first union shows that $\bar{A}^{\mathcal{C}}$ is closed and the second that it is $\mathcal{C}$-constructible. One may easily check that $\bar{A}^{\mathcal{C}}$ satisfies the required minimality property.

Define $Z:=\bar{A}^{Z}$, where $\bar{A}^{Z}$ denote the Zariski closure of $A$. Replacing $X$ by $X \cap Z$, if necessary, we may suppose $A \subset X \subset Z$ and $Z=\bar{X}^{Z}$. There is an algebraic subset $S \subset Z$ such that $\operatorname{dim} S<\operatorname{dim} Z, Z \backslash S$ is a topological manifold of pure dimension, and $X \backslash S$ is the union of some connected components of $Z \backslash S$. To each $C \subset X, C$ closed in $X$, $\mathcal{C}$-constructible and containing $A$, we associate the set of connected components of $X \backslash S$ that are entirely contained in $C$. Taking the intersection of finitely many such $C$ we find such a set, denoted $C_{0}$, that has minimal number of such components. Let $A_{1}$ denote the union of these components. Thus all closed in $X, \mathcal{C}$-constructible sets containing $A$ contain $A_{1}$.

Let $X^{\prime}=(X \cap S) \cup C_{0} . X^{\prime}$ is $\mathcal{C}$-constructible and closed in $X$. By lemma 1.6, $\operatorname{dim}\left(C_{0} \backslash\right.$ $\left.\left(A \cup A_{1}\right)\right)<\operatorname{dim} C_{0}=\operatorname{dim} X$ and hence $\operatorname{dim}\left(X^{\prime} \backslash\left(A \cup A_{1}\right)\right)<\operatorname{dim} X^{\prime}=\operatorname{dim} X$. Then the $\mathcal{C}$-closure of $A \cup A_{1}$ in $X^{\prime}$, that exists by the first case considered above, is the $\mathcal{C}$-closure of $A$ in $X$. This ends the proof.

Remark 2.6. Clearly $\operatorname{dim} \bar{A}^{\mathcal{C}}=\operatorname{dim} A$. If $A$ is $\mathcal{C}$-constructible then $\bar{A}^{\mathcal{C}}=A \cup \overline{(\bar{A} \backslash A)}$ and hence $\operatorname{dim}\left(\bar{A}^{\mathcal{C}} \backslash A\right)<\operatorname{dim} A$.

Theorem 2.7. Algebraically constructible sets form a constructible category.

This follows by standard argument from theorems 2.1 and 2.2 but we postpone the proof until section 4 where we deduce it from the topological properties of algebraically constructible functions (a formal approach to the integration with respect to the Euler characteristic on real algebraic sets). Let us just note that A1 and A2 are satisfied trivially and A4 follows from theorem 2.1 by the additivity of the Euler characteristic with compact supports (on the link). A3 for regular mappings is easy (we have showed already A3 (b)).

In particular there is the algebraically constructible closure. Let $A$ be a semi-algebraic subset of a real algebraic set $X$. Then there exists the smallest set, denoted by $\bar{A}^{\mathcal{A C}}$, closed and algebraically constructible in $X$ that contains $A$. Clearly $\bar{A} \subset \bar{A}^{\mathcal{A C}} \subset \bar{A}^{Z}$, where $\bar{A}^{Z}$ denote Zariski closure of $A$, but each inclusion may be strict. For instance if $A$ is the regular part of the Whitney umbrella $\left\{z x^{2}=y^{2}\right\} \subset \mathbb{R}^{3}$ then $\bar{A}^{\mathcal{A C}}=\bar{A}^{Z}$ and is strictly bigger than $\bar{A}$. If $A$ is the regular part of the Cartan umbrella $\left\{z\left(x^{2}+y^{2}\right)=x^{3}\right\} \subset \mathbb{R}^{3}$ then $\bar{A}=\bar{A}^{\mathcal{A C}}$ but is strictly smaller than $\bar{A}^{Z}$. 


\section{Proof of Borel-Kurdyka Theorem}

First we recall the theorem of Borel [7] restated for constructible categories.

Theorem 3.1. Let $X$ be a locally compact semi-algebraic set belonging to a constructible category $\mathcal{C}$. Let $f: X \rightarrow X$ be an injective continuous $\mathcal{C}$-map. If $f$ is open then it is a homeomorphism. In particular such $f$ is surjective.

The original theorem of Borel is stated for non-singular real algebraic sets and injective regular maps. Such maps are open by the invariance of domain, cf. [15] (one may use alternatively corollary 1.7). The openess is also guaranteed if $X$ is a normal complex algebraic variety and $f: X \rightarrow X$ is an injective regular morphism of complex algebraic varieties. Then passing to normalization Borel shows that any injective regular self-mapping of a complex algebraic variety is surjective.

We shall recall below the main idea of [7]. The existence of the fundamental class in the Borel-Moore homology with $\mathbb{Z}_{2}$ coefficients plays the crucial role in this argument. Let $d=\operatorname{dim}(X \backslash f(X))$. Denote $f^{k+1}=f \circ f^{k}, f^{0}=\operatorname{id}_{X}$, and $Y_{k}=X \backslash f^{k}(X)$. The sets $Y_{k}$ are closed in $X$ and Euler in codimension 1, cf. remark 1.5. The set $Y_{\ell} \backslash Y_{\ell-1}$ are all disjoint and of dimension $d$ and hence $\left[Y_{\ell}\right]-\left[Y_{\ell-1}\right]=\left[Y_{\ell} \backslash Y_{\ell-1}\right], \ell=1, \ldots, k$, are linearly independent in $H_{d}^{B M}\left(Y_{k}, \mathbb{Z}_{2}\right)$. Thus

$$
\operatorname{dim} H_{d}^{B M}\left(Y_{k}, \mathbb{Z}_{2}\right) \geq k
$$

Note that $f^{k}: X \rightarrow f^{k}(X)$ is a homeomorphism and $f^{k}(X)$ is open in $X$. Thus the long exact sequence (1.2),

$$
\longrightarrow H_{d+1}^{B M}\left(f^{k}(X), \mathbb{Z}_{2}\right) \longrightarrow H_{d}^{B M}\left(Y_{k}, \mathbb{Z}_{2}\right) \longrightarrow H_{d}^{B M}\left(X, \mathbb{Z}_{2}\right) \longrightarrow,
$$

gives a bound independent on $k$

$$
\operatorname{dim} H_{d}^{B M}\left(Y_{k}, \mathbb{Z}_{2}\right) \leq \operatorname{dim} H_{d}^{B M}\left(X, \mathbb{Z}_{2}\right)+\operatorname{dim} H_{d+1}^{B M}\left(X, \mathbb{Z}_{2}\right),
$$

that contradicts (3.1).

Theorem 3.2. Let $X$ be a locally compact semi-algebraic set belonging to a constructible category $\mathcal{C}$. Let $f: X \rightarrow X$ be a continuous morphism in $\mathcal{C}$ that is injective as a map. Then $f$ is surjective.

By theorems 2.7 and 4.5 , proven in the next sections, we get the following.

Corollary 3.3. (Borel-Kurdyka Theorem) Any injective regular self-mapping of a real algebraic variety is surjective.

Corollary 3.4. (cf. [11]) Any injective continuous self-mapping of a locally compact arc-symmetric set, the graph of which is arc-symmetric, is surjective.

Proof of theorem 3.9. We proceed by induction on $n=\operatorname{dim} X$. Let $\Sigma=\operatorname{Sing}(X)$ be the set of topological singularities of $X$. That is $\Sigma$ is the disjoint union of the following sets

$$
\begin{aligned}
S & =\left\{x \in X ; \operatorname{dim}_{x} X=n,(X, x) \text { not homeomorphic to }\left(\mathbb{R}^{n}, 0\right)\right\}, \\
A & =\left\{x \in X ; \operatorname{dim}_{x} X<n,\right\} .
\end{aligned}
$$

By the existence of locally topologically trivial semi-algebraic stratification of $X$ both $A$ and $S$ are semi-algebraic. It is easy to see that $S$ and $\Sigma=S \cup A$ are closed in $X$. We shall show that

$$
f\left(\bar{\Sigma}^{\mathcal{C}}\right) \subset \bar{\Sigma}^{\mathcal{C}} .
$$


By lemma 1.6, $f(S) \subset S$. Therefore, by the lemma below, $f\left(\bar{S}^{\mathcal{C}}\right) \subset \bar{S}^{\mathcal{C}}$.

Lemma 3.5. Let $B \subset X$ such that $f(B) \subset B$. Then $f\left(\bar{B}^{\mathcal{C}}\right) \subset \bar{B}^{\mathcal{C}}$.

Proof. $f(B) \subset B$ gives

$$
B \subset f^{-1}(f(B)) \subset f^{-1}(B) \subset f^{-1}\left(\bar{B}^{\mathcal{C}}\right) .
$$

Note that $f^{-1}\left(\bar{B}^{\mathcal{C}}\right)$ is closed and in $\mathcal{C}$. Hence (3.3) gives $\bar{B}^{\mathcal{C}} \subset f^{-1}\left(\bar{B}^{\mathcal{C}}\right)$. That is $f\left(\bar{B}^{\mathcal{C}}\right) \subset$ $f\left(f^{-1}\left(\bar{B}^{\mathcal{C}}\right)\right) \subset \bar{B}^{\mathcal{C}}$ as required.

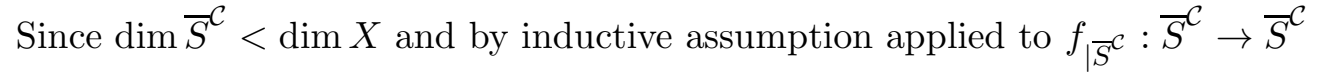

$$
f\left(\bar{S}^{\mathcal{C}}\right)=\bar{S}^{\mathcal{C}} \text {. }
$$

Let $U=X \backslash \bar{\Sigma}^{\mathcal{C}}$. We shall show $f(U) \subset U$. Firstly, by (3.4) and the definition of $A$, $f(U) \subset X \backslash\left(A \cup \bar{S}^{\mathcal{C}}\right)$. Since $A \cup \bar{S}^{\mathcal{C}}$ is closed in $X, X \backslash\left(A \cup \bar{S}^{\mathcal{C}}\right)$ is open in $X$. By Lemma 1.7, $f(U)$ is open in $X \backslash\left(A \cup \bar{S}^{\mathcal{C}}\right)$ and hence in $X$. Consequently $X \backslash f(U)$ is closed in $X$ and, by axiom A3 (b), $\mathcal{C}$-constructible. Thus, since $A \cup \bar{S}^{\mathcal{C}} \subset X \backslash f(U)$, by the definition of $\mathcal{C}$-closure, $\bar{\Sigma}^{\mathcal{C}}=\bar{A}^{\mathcal{C}} \cup \bar{S}^{\mathcal{C}} \subset X \backslash f(U)$. That is $f(U) \subset U$ as claimed. Since $U$ is a $\mathcal{C}$-constructible topological manifold and $f_{\mid U}: U \rightarrow U$ is injective, it is surjective by Theorem 3.1. This means $f(U)=U$ and shows (3.2). By the inductive assumption, $f\left(\bar{\Sigma}^{\mathcal{C}}\right)=\bar{\Sigma}^{\mathcal{C}}$, and therefore $f(X)=X$ as claimed.

We show in section 5 below that $f$ is a homeomorphism. This will give in particular that $f(A)=A, f(S)=S$, and $f(\Sigma)=\Sigma$.

\section{Examples of Constructible Categories}

4.1. Algebraically Constructible Sets. The algebraically constructible sets, see definition 2.3, can be studied using the theory of algebraically constructible functions, see [13]. Let $Z$ be a real algebraic set. An algebraically constructible function on $Z$ is an integer-valued function which can be written as a finite sum

$$
\varphi=\sum m_{i} f_{i *} \mathbf{1}_{Z_{i}}
$$

where for each $i, Z_{i}$ is an algebraic set, $\mathbf{1}_{Z_{i}}$ is the characteristic function of $Z_{i}, f_{i}: Z_{i} \rightarrow Z$ is a proper regular map, and $m_{i}$ is an integer.

Theorem 4.1. Let $X$ be a semi-algebraic subset of $\mathbb{R}^{N}$. The following conditions are equivalent:

(i) $X$ is an algebraically constructible set.

(ii) The characteristic function $\mathbf{1}_{X}$ of $X$ is an algebraically constructible on $\mathbb{R}^{N}$.

(iii) There exist polynomials $g_{1}, \ldots, g_{k} \in \mathbb{R}\left[x_{1}, \ldots, x_{N}\right]$ such that $\mathbf{1}_{X}=\sum \operatorname{sign} g_{i}$.

(iv) There exists a real algebraic morphism $F: Z \rightarrow \mathbb{R}^{N}$ such that

$$
\begin{aligned}
& x \in X \text { iff } \chi\left(F^{-1}(x)\right)=1 \\
& x \notin X \text { iff } \chi\left(F^{-1}(x)\right)=0 .
\end{aligned}
$$

(v) There exists a real algebraic morphism $F: Z \rightarrow \mathbb{R}^{N}$ such that

$$
\begin{array}{ll}
x \in X \text { iff } \chi\left(F^{-1}(x)\right) \equiv 1 & (\bmod 2) \\
x \notin X \text { iff } \chi\left(F^{-1}(x)\right) \equiv 0 & (\bmod 2) .
\end{array}
$$


Proof. (i) implies (ii) by the definition of algebraically constructible functions. (ii) is equivalent to (iii) by [17] or [18]. (ii) (resp. (iii)) implies (iv) by [13] (resp. [17]). (iv) implies (v) trivially. Finally (v) implies (i) follows easily from Theorem 2.2.

Proof of theorem 2.7. We show it only for subsets of $\mathbb{R}^{N}$. The proof for the subsets of $\mathbb{R} \mathbb{P}^{N}$ is similar.

Axioms A1 and A2 are satisfied trivially. To show A3 we use the fact that the algebraically constructible functions are stable by the inverse image and the push forward by regular maps, see [13]. Let $f: X \rightarrow Y$ be a map between two algebraically constructible subsets of $\mathbb{R}^{n}$ and $\mathbb{R}^{m}$ respectively and suppose that the graph of $f, \Gamma_{f} \subset \mathbb{R}^{n} \times \mathbb{R}^{m}$, belongs to $\mathcal{A C}$. Denote the projections of $\Gamma_{f}$ on $X$ and $Y$ by $\pi_{X}$ and $\pi_{Y}$ respectively. Let $B \subset Y, B \in \mathcal{A C}$. Then $\pi_{Y}^{-1}(B) \in \mathcal{A C}$ and hence the characteristic function of $\pi_{Y}^{-1}(B)$ is algebraically constructible. Consequently its push-forward onto $X$ by $\pi_{X}$, that equals the characteristic function of $f^{-1}(B)$, is algebraically constructible. This shows that $f^{-1}(B) \in \mathcal{A C}$ and $\mathrm{A} 3$ (a) is shown. If $A \subset X, A \in \mathcal{A C}$, and $\left.f\right|_{A}$ is injective then $\pi_{Y}$ restricted to $\pi_{X}^{-1}(A)$ is injective and the same argument as above shows that $f(A)=\pi_{Y}\left(\pi_{X}^{-1}(A)\right) \in \mathcal{A C}$. This ends the proof of $\mathrm{A} 3$.

A4 follows from the fact the link of an algebraically constructible function is even 13. theorem 2.5.

4.2. Arc-Symmetric Sets. We call $X \subset \mathbb{R P}^{N}$ arc-symmetric if for every real analytic arc $\gamma(t):(-\varepsilon, \varepsilon) \rightarrow \mathbb{R P}^{N}$ such that $\gamma((-\varepsilon, 0)) \subset X$ there is $\varepsilon^{\prime}>0$ such that $\gamma\left(\left(0, \varepsilon^{\prime}\right)\right) \subset X$. A subset $X \subset \mathbb{R}^{N}$ is called arc-symmetric if it is arc-symmetric as a subset of $\mathbb{R P}^{N}$. If $X$ is arc-symmetric then we write $X \in \mathcal{A S}$ for short.

Remark 4.2. The arc-symmetric sets were first introduced and studied by Kurdyka in [10] but his original notion of arc-symmetric sets differs sligthly from ours. In [10] Kurdyka considers only closed semi-algebraic subsets $\mathbb{R}^{N}$ such that for every real analytic arc $\gamma(t)$ : $(-\varepsilon, \varepsilon) \rightarrow \mathbb{R}^{N}$ if $\gamma((-\varepsilon, 0)) \subset X$ then $\gamma((-\varepsilon, \varepsilon)) \subset X$. Note that this definition does not give a sufficent control of the sets in question at infinty. For instance one branch of a hyperbola is arc-symmetric in the sense of Kurdyka but not in ours. Its projective compactification is not arc-symmetric neither in Kurdyka's sense nor in ours. But for the proof of surjectivity theorem, that is of Corollary 3.3, Kurdyka uses in [11] the sets that remain arc-symmetric (in his sense) after compactification of $\mathbb{R}^{N}$ that is in our terminology precisely the arc-symmetric subsets of $\mathbb{R} \mathbb{P}^{N}$ contained and closed in $\mathbb{R}^{N}$. Kurdyka's arcsymmetric sets are Euler, cf. [13], but they do not form a constructible category.

The categories of algebraically constructible sets and arc-symmetric sets are very similar. The topological properties of arc-symmetric sets can be studied by means of Nashconstructible functions [13], [6], a tool analogous to algebraically constructible functions. The following theorem shows these similarities. It is simpler to us to formulate it for subsets of projective spaces so we avoid the passage to compactification.

Theorem 4.3. Let $X$ be a semi-algebraic subset of $\mathbb{R P}^{N}$. The following conditions are equivalent:

(i) $X$ is arc-symmetric.

(ii) $\mathbf{1}_{X}$ is Nash constructible as a function (see [13]).

(iii) There exist blow Nash functions (see [6]) $g_{1}, \ldots, g_{k}$ such that $\mathbf{1}_{X}=\sum \operatorname{sign} g_{i}$. 
(iv) There exists a real algebraic morphism $F^{\prime}: Z^{\prime} \rightarrow \mathbb{R P}^{N}$ and $Z$ a union of connected components of $Z$ such that for $F=F_{\mid Z}^{\prime}$

$$
\begin{aligned}
& x \in X \text { iff } \chi\left(F^{-1}(x)\right)=1 \\
& x \notin X \text { iff } \chi\left(F^{-1}(x)\right)=0 .
\end{aligned}
$$

(v) There exists a real algebraic morphism $F^{\prime}: Z^{\prime} \rightarrow \mathbb{R P}^{N}$ and $Z$ a union of connected components of $Z^{\prime}$ such that for $F=F_{\mid Z}^{\prime}$

$$
\begin{aligned}
& x \in X \text { iff } \chi\left(F^{-1}(x)\right) \equiv 1 \quad(\bmod 2) \\
& x \notin X \text { iff } \chi\left(F^{-1}(x)\right) \equiv 0 \quad(\bmod 2) \text {. }
\end{aligned}
$$

Proof. (i) is equivalent to (ii) by the argument of section 5 of [13]. (ii) is equivalent to (iii) by [6]. (ii) implies (iv) by [13]. (iv) implies (v) trivially.

We show (v) implies (i). For this we note that each closed arc-symmetric subset of $\mathbb{R P}^{N}$ can be written uniquely as a finite union of irreducible closed arc-symmetric sets, see [10]. On the other hand we have after [13].

Lemma 4.4. Let $F^{\prime}: Z^{\prime} \rightarrow \mathbb{R P}^{N}$ be a morphism of real algebraic varieties, $Z \subset Z^{\prime}$ closed and arc-symmetric. Denote $F=F_{\mid Z^{\prime}}^{\prime}$ and suppose that $F(Z) \subset Y$, where $Y$ is closed arc-symmetric and irreducible. Then there exists a proper arc-symmetric subset $Y^{\prime} \subset Y$, $\operatorname{dim} Y^{\prime}<\operatorname{dim} Y$, such that $\chi\left(F^{-1}(x)\right)$ is constant modulo 2 on $Y \backslash Y^{\prime}$.

The rest of proof is similar to the algebraically constructible case.

Theorem 4.5. Arc-symmetric sets form a constructible category.

Proof. It follows from the topological properties of Nash-constructible functions as developed in [13] section 5. First the links of Nash constructible functions are even that shows A4. To show A3 we need to show the Nash constructible functions are stable by inverse image and pushforward by mappings with arc-symmetric graph. It is clear from [13] section 5 the Nash constructible functions are stable by inverse image and pushforward by proper regular maps. Let $f: X \rightarrow Y, X \subset \mathbb{R P}^{n}, Y \subset \mathbb{R P}^{m}$, where $X, Y$ and the graph $\Gamma$ of $f$ are arc-symmetric. We denote the projections of $\mathbb{R} \mathbb{P}^{n} \times \mathbb{R P}^{m}$ to $\mathbb{R} \mathbb{P}^{n}$ and $\mathbb{R P}^{m}$ by $\pi_{n}$ and $\pi_{m}$ respectively. Let $\varphi$, resp. $\psi$, be a Nash constructible function supported in $X$, resp. $Y$. On the level of constructible functions the restiction to $\Gamma$ correponds to the multiplication by $\mathbf{1}_{\Gamma}$ and hence $f_{*}(\varphi)=\left(\pi_{m}\right)_{*}\left(\left(\pi_{n}\right)^{*} \cdot \mathbf{1}_{\Gamma}\right), f^{*}(\psi)=\left(\pi_{n}\right)_{*}\left(\left(\pi_{m}\right)^{*} \cdot \mathbf{1}_{\Gamma}\right)$.

The rest of the proof is completely analogous to the proof of theorem 2.7 .

4.3. Remarks. Clearly $\mathcal{A C}$ is the smallest constructible category. The difference between $\mathcal{A C}$ and $\mathcal{A S}$ lies in the fact that a compact connected component of an arc-symmetric set is arc-symmetric.

Proposition 4.6. Every constructible category is contained in $\mathcal{A S}$. Furthermore, $\mathcal{A S}$ is the only constructible category containing the connected components of compact real algebraic sets.

Proof. Let $\mathcal{C}$ be a constructible category and let $X \subset \mathbb{R P}^{N}$ be a $\mathcal{C}$-constructible set. We show that $X$ is arc-symmetric by induction on $\operatorname{dim} X$. Let $\operatorname{dim} X=n$ and suppose first that $X$ is closed in $\mathbb{R} \mathbb{P}^{N}$. Consider

$$
\pi: \widetilde{Y} \rightarrow Y
$$


a resolution of $Y=\bar{X}^{Z}$. Let $S \subset Y$ be an algebraic subset of $Y$ such that $Y \backslash S$ is regular of pure dimension $n, \operatorname{dim} S<n$, and

$$
\widetilde{\pi}:=\pi_{\mid}: \tilde{Y} \backslash \pi^{-1}(S) \rightarrow Y \backslash S
$$

is an isomorphism. Denote $\widetilde{S}:=\pi^{-1}(S) . \pi^{-1}(X)$ is a $\mathcal{C}$-constructible subset of $\tilde{Y}$ and hence by lemma 1.6

$$
\pi^{-1}(X)=X_{1} \cup X_{2},
$$

where $X_{1}$ is the union of some connected components of $\widetilde{Y}$ and $\operatorname{dim} X_{2}<n$. Note that $X^{\prime}=\pi\left(X_{1}\right) \cup S$ is arc-symmetric, see [10] for details. Let $S^{\prime}=S \cup{\overline{\pi\left(X_{2}\right)}}^{Z}$. Then, by the above $X \backslash S^{\prime}=X^{\prime} \backslash S^{\prime}$ is arc symmetric. On the other hand, $X \cap S^{\prime}$ is $\mathcal{C}$-constructible and hence arc symmetric by the inductive assumption. This shows that $X$ is arc-symmetric.

Consider $\mathcal{C}$-constructible $X \subset \mathbb{R P}^{N}$, not necessarily closed in $\mathbb{R P}^{N}$. Then, by Remark 2.6, $\operatorname{dim}\left(\bar{X}^{\mathcal{C}} \backslash X\right)<\operatorname{dim} X . \bar{X}^{\mathcal{C}}$ is closed in $\mathbb{R P}^{N}$ and hence arc-symmetric by the above and $\bar{X} \backslash X$ is arc-symmetric by the inductive assumption. This shows that $X$ is arc-symmetric and hence the first claim of proposition.

Suppose now that $\mathcal{C}$ is a constructible category containing the connected components of compact real algebraic sets. Let $X \subset \mathbb{R P}^{N}$ be closed and arc-symmetric. We show that $X \in \mathcal{C}$. Apply to $X$ the construction of the first part of proof. We find again (4.2), where now $X_{1} \in \mathcal{C}$. Hence, be axiom A3, so does $\pi\left(X_{1} \backslash \widetilde{S}\right)=X \backslash S^{\prime}$, where again $S^{\prime}=S \cup{\overline{\pi\left(X_{2}\right)}}^{Z}$. Again, $X \cap S^{\prime}$ is arc-symmetric and of dimension $<\operatorname{dim} X$ and hence belongs to $\mathcal{C}$ by the induction on dimension. This shows that $X=(X \backslash S) \cup\left(X \cap S^{\prime}\right)$ is in $\mathcal{C}$ as required.

By [13] we have the following strenghtening of axiom 4 .

Corollary 4.7. Every locally compact $\mathcal{C}$-constructible set is Euler.

\section{Hierarchy OF SINGUlarities}

We say that two germs $(Y, y)$ and $(X, x)$ of locally compact semi-algebraic sets are semi-algebraically homeomorphic, and write $(Y, y) \sim(X, x)$, if there exists the germ of a semi-algebraic homeomorphism $f:(Y, y) \rightarrow(X, x)$.

Let $X$ be a locally compact semi-algebraic set. Then there is a semi-algebraic stratification of $X$ such that $(X, y) \sim(X, x)$ if $y, x$ belong to the same stratum. Indeed, this follows from from the existence of a semi-algebraic triangulation of $X$. Moreover, by the same argument, this stratification can be chosen locally topologically trivial (i.e. for any stratum $S$ and any $x \in S$ there is a neighbourhood $U$ of $x$ in $X$ and a continuous retraction $\pi: U \rightarrow S \cap U,\left.\pi\right|_{S \cap U}=i d_{S \cap U}$, such that $\pi$ is a trivial fibration). For each $x \in X$ consider

$$
S_{x}=\{y \in X ;(X, y) \sim(X, x)\} .
$$

Clearly $S_{x}$ is a union of strata so it is semi-algebraic. Consequently at its generic point

$S_{x}$ is a topological manifold. But the germ of $X$ at arbitrary point of $S_{x}$ is homeomorphic to that at a generic point of $S_{x}$. Thus we get

Proposition 5.1. Each $S_{x}$ is a locally closed semi-algebraic subset of $X$ and a puredimensional topological manifold. The decomposition into the equivalence classes of semialgebraic homeomorphisms induces a canonical, locally topologically trivial, (topological) stratification of $X$. 
We call this stratification the stratification by s.-a. topological types.

From now on we restrict ourselves to Euler locally compact semi-algebraic sets. We denote by $\mathcal{G}$ the set of equivalence classes of such sets divided by semi-algebraic homeomorphisms.

Definition 5.2. Let $(Y, y)$ and $(X, x)$ be two germs of Euler locally compact semi-algebraic sets. We write $(Y, y) \prec(X, x)$ if there exists the germ of a continuous semi-algebraic injective map $i:(Y, y) \rightarrow(X, x)$.

Proposition 5.3. If $(Y, y) \prec(X, x)$ and $(X, x) \prec(Y, y)$ then $(Y, y)$ and $(X, x)$ are semialgebraically homeomorphic. Thus $\prec$ gives a partial order on $\mathcal{G}$.

Proof. $\prec$ is clearly transitive. If $(X, x) \prec(Y, y)$ and $(Y, y) \prec(X, x)$ then $(X, x) \prec(X, x)$ that is there exists a continuous semialgebraic injective map $i:(X, x) \rightarrow(X, x)$ that must be a homeomorphism by proposition 1.3 (ii).

Note that after Lemma 1.6 if $X$ is a topological manifold at $x$ of dimension $n$ and $(Y, y) \prec(X, x)$, with $\operatorname{dim}_{y} Y=n$, then $(Y, y) \sim(X, x)$. In a way $(Y, y) \prec(X, x)$ means that the singularity of $Y$ at $y$ is less complicated then that of $X$ at $x$ (at least if $\operatorname{dim}_{y} Y=$ $\left.\operatorname{dim}_{x} X\right)$. We want that the singularities around $x$ are not more complicated than that at $x$.

Proposition 5.4. Let $X$ be an Euler locally compact semi-algebraic set and let $x \in X$. If $x \in \overline{S_{y}}$ and $(X, x) \prec(X, y)$ then $(X, x) \sim(X, y)$.

Proof. The proof is by induction on the codimension of $S_{y}$ in $X$. If this codimension is zero then $X$ is a topological manifold at $y$ and the result follows from corollary 1.7.

In the general case suppose that there exists a continuous semi-algebraic injection $f$ : $(X, x) \rightarrow(X, y)$. Choosing $y^{\prime} \in S_{y}$ arbitrarily close to $x$ we get $z=f\left(y^{\prime}\right)$ arbitrarily close to $y$. That is $y \in \overline{S_{z}}$ and $(X, y) \prec(X, z)$. Note that $y \in \overline{S_{z}}$ gives $S_{y} \subset \overline{S_{z}}$, in particular, $\operatorname{dim} S_{z}>\operatorname{dim} S_{y}$. Therefore, by the inductive assumption, $(X, y) \sim(X, z)$.

Lemma 5.5. Let $X$ be an Euler locally compact semi-algebraic set, $S \subset X$ a connected component of a stratum of the stratification of $X$ by s.-a. topological types. Let $Y \subset X$ be Euler closed semi-algebraic. Then, if

$$
(Y, x)=(X, x)
$$

holds for an $x \in S$ then it does so for all $x \in S$.

Proof. The set $S_{1}=\{x \in S ;(Y, x)=(X, x)\}$ is clearly open in $S$. We show that it is closed.

Denote by $X_{\text {reg }}^{d}$ the set of points of $X$ at which $X$ is a topological manifold of dimension $d$. Then

$$
Y_{r e g}^{d} \cap X_{r e g}^{d}
$$

is open and closed in $X_{\text {reg }}^{d}$. Indeed it is clearly open. Suppose $x \in \overline{Y_{r e g}^{d} \cap X_{r e g}^{d}} \cap X_{r e g}^{d}$. Then $x \in Y$ since $Y$ is closed in $X$ and $\operatorname{dim}_{x} Y$ must be $d$. Hence $x \in Y_{\text {reg }}^{d}$ by lemma 1.6 . This shows the claim.

Let $x \in \bar{S}_{1} \cap S$. Fix an integer $d$ and let $X^{\prime}$ be a connected component of $X_{\text {reg }}^{d}$ such that $x \in \overline{X^{\prime}}$. Hence, by local topological triviality of stratification, there is a neighbourhood $U_{S}$ of $x$ in $S$ such that $U_{S} \subset \overline{X^{\prime}} . X^{\prime} \cap Y_{\text {reg }}^{d}$ is nonempty since $U_{S} \cap S_{1} \neq \emptyset$. Thus, by the above claim, $X^{\prime} \cap Y_{\text {reg }}^{d}=X^{\prime}$ and hence $\overline{X^{\prime}} \subset Y$. Since the union of such $\overline{X^{\prime}}$ form a neighbourhood of $x$ in $X$ we get $(X, x)=(Y, x)$ as claimed. 
We complete the proof of proposition 5.4. Let $B_{y}$ a small open semi-algebraic neighbourhood (e.g. a ball) of $y$ in $X$ and let $B_{x}=f^{-1}\left(B_{y}\right)$. Then the restriction of $f, B_{x} \rightarrow B_{y}$ is injective and its image $f\left(B_{x}\right)$ is a closed semi-algebraic and Euler subset of $B_{y}$. We apply lemma 5.5 to $f\left(B_{x}\right) \subset B_{y}$ and the stratum $S=S_{y} \cap B_{y}$. Recall that there exists $z \in S, z=f\left(y^{\prime}\right)$, where $y^{\prime} \in S_{y}$ is close to $x$, such that $(X, y) \sim(X, z)$. Then $f$ is a homeomorphism at $y^{\prime}$ by proposition $1.3(\mathrm{~b})$. Thus $\left(f\left(B_{x}\right), z\right)=\left(B_{y}, z\right)$ and by lemma 5.5 $\left(f\left(B_{x}\right), y\right)=\left(B_{y}, y\right)$. That is $f$ is a local homeomorphism at $x$. This ends the proof.

As a corollary we obtain the following

Theorem 5.6. Let $X$ be a locally compact Euler semi-algebraic set of pure dimension $n$. Let $f: X \rightarrow X$ be continuous and semi-algebraic. If $f$ is injective and surjective then it is a homeomorphism.

Proof. Let $\mathcal{S}$ be the stratification of $X$ by semi-algebraic topological types. It suffices to show that for each stratum $S \in \mathcal{S}$ we have $f(S)=S$. Indeed, then, by proposition 1.3 (b), $f$ is a local homeomorphism at every $x \in X$ and the theorem follows. The proof is by induction on number of strata in $\mathcal{S}$. If there is only one type, that is $X$ is a topological manifold, then the result follows from the invariance of domain or corollary 1.7.

Let $S$ be a stratum of $\mathcal{S}$ of maximal singularity type (with respect to $\prec$ ). Then $f(S) \subset S$. By lemma 1.6, $f(S)$ is open in $S$. We show that $f(S)$ is also closed in $S$. By propositions 5.1 and 5.4, $S$ is a topological manifold closed in $X$. Consider the restriction of $f, f^{-1}(S) \rightarrow S$. Let $y \in S, f(x)=y$, and choose, as in the proof of proposition 5.4, $B_{y}$ a small open semialgebraic neighbourhood of $y$ in $X$ and let $B_{x}=f^{-1}\left(B_{y}\right)$. Then, see the proof of lemma 5.5, $Y^{\prime}=\left(f\left(B_{x}\right)\right)_{\text {reg }} \cap\left(B_{y}\right)_{\text {reg }}$ is open and closed in $\left(B_{y}\right)_{\text {reg }}$. We have $y \in \overline{Y^{\prime}}$ and hence, by local topological triviality of stratification $\mathcal{S}, S \cap B_{y} \subset \overline{Y^{\prime}}$. This shows that the mapping $f^{-1}(S) \rightarrow S$ is open. Consequently, since $S$ is closed in $X, S \backslash f(S)=f\left(f^{-1}(S) \cap(X \backslash S)\right)$ is open in $S$. Thus we have showed that $f(S)$ is open and closed in $S$ that is a union of connected components of $S$. Corollary 1.7 shows that the restriction of $f, S \rightarrow f(S)$ is a homeomorphism, in particular $S$ and $f(S)$ are homeomorphic. Hence $f(S)=S$.

To complete the proof of theorem we apply the inductive assumption to $\left.f\right|_{X \backslash S}: X \backslash S \rightarrow$ $X \backslash S$.

Theorem 5.7. Let $X$ be a locally compact $\mathcal{C}$-constructible semi-algebraic set and let $f$ : $X \rightarrow X$ be an injective continuous $\mathcal{C}$-constructible map. Then $f$ is a homeomorphism.

Proof. $f$ is surjective by theorem 3.2. Let $\operatorname{dim} X=n$ and denote by $\mathcal{S}$ be the stratification of $X$ by semi-algebraic topological types. As in the proof of theorem 5.6 it suffices to show that $f(S)=S$ for each stratum $S$.

Lemma 5.8. There is a filtration of $X$ by closed in $X, \mathcal{C}$-constructible, and stable by $f$ sets

$$
X=X^{n} \supset X^{n-1} \supset \cdots \supset X^{0} \supset X^{-1}=\emptyset
$$

such that for every $i: \dot{X}^{i}:=X^{i} \backslash X^{i-1}$ is a topological manifold of pure dimension $i$ (or empty) and $(X, y) \sim(X, x)$ for all $y, x$ belonging to the same connected component of $\dot{X}^{i}$.

(stability by $f$ for $X^{i}$ means $f\left(X^{i}\right) \subset X^{i}$. By theorem 3.9 it is equivalent to $f\left(X^{i}\right)=$ $X^{i}$.)

Proof. By descending induction on $k$ we construct a filtration

$$
X=X^{n} \supset X^{n-1} \supset \cdots \supset X^{k}
$$


satisfying all the above properties except that $X^{k}$ can be arbitrary closed in $X, \mathcal{C}$ constructible, and stable by $f$ set of dimension at most $k$. If $k=n-1$ then we take as $X^{n-1}$ the $\mathcal{C}$-closure of the singularities of $X$, i. e. the set $\tilde{\Sigma}$ of the proof of theorem 3.3 . Suppose that the filtration (5.1) has been already constructed. We construct $X^{k-1}$. Set

$$
Y=\bigcup_{S \in \mathcal{S}} \Sigma\left(S \cap X^{k}\right)
$$

where $\Sigma\left(S \cap X^{k}\right)$ denote the set of points where $S \cap X^{k}$ is not topological manifold of dimension $k$. Then $X^{k} \backslash Y$ is a topological manifold of dimension k. Let

$$
X^{k-1}=\bar{Y}^{\mathcal{C}} \text {. }
$$

It is clear that $X^{k-1}$ satisfies the required properties.

Fix an integer $i$. By construction $f$ sends the connected components of $\dot{X}^{i}$ onto the connected components of $\dot{X}^{i}$. To each such connected component corresponds one singularity type. Thus the components corresponding to the maximal types (with respect to $\prec)$ are sent onto the components of the same type. Hence, by inductive argument on the number of types (or components), we see that for each stratum $S, f\left(S \cap \dot{X}^{i}\right)=\left(S \cap \dot{ }^{i}\right)$. This, for all $i$, shows $f(S)=S$. This ends the proof of theorem.

Corollary 5.9. Any injective regular self-mapping of a real algebraic variety is a homeomorphism.

Corollary 5.10. Any injective continuous self-mapping of a locally compact arc-symmetric set, the graph of which is arc-symmetric, is a homeomorphism.

\section{REFERENCES}

[1] S. Akbulut, H. King, The topology of real algebraic sets, Enseign. Math. 29 (1983), 221-261

[2] E. Akin, Stiefel-Whitney homology classes and bordism, Trans. A.M.S. 205 (1975), 341-359

[3] J. Ax, Injective endomorphisms of varieties and schemes, Pac. J. Math. 31 no. 1 (1969), 1-17

[4] J. Bochnak, M. Coste, M.-F. Roy, Géométrie algébrique réelle Springer-Verlag, Berlin 1987

[5] A. Białynicki-Birula, M. Rosenlicht, Injective morphisms of real algebraic varieties, Proc. Amer. Math. Soc. 13 (1962), 200-203

[6] I. Bonnard, Nash constructible functions, Université d'Angers preprint No. 128, Mars 2001

[7] A. Borel, Injective endomorphisms of algebraic Nash varieties, Arch. Math. 20 (1969), 531-537

[8] A. Borel, J.C. Moore, Homology theory for locally compact spaces, Michigan Math. J. 7 (1960), 137-159

[9] M. Gromov, Endomorphisms of symbolic algebraic varieties, J. Eur. Math. Soc. 1, 109-197

[10] K. Kurdyka, Ensembles semi-algébriques symétriques par arcs, Math. Ann. 281 no.3 (1988), 445-462

[11] K. Kurdyka, Injective endomorphisms of real algebraic sets are surjective, Math. Ann. 313 no.1 (1999), 69-83

[12] K. Kurdyka, K. Rusek, Surjectivity of Certain Injective Semi-algebraic Tranformations of $\mathbb{R}^{n}$, Math. Z. 200 no.1 (1988), 141-148

[13] C. McCrory, A. Parusiński, Algebraically constructible functions, Annales Sci. Ec. Norm. Sup. 30:4 (1997), 527-552.

[14] C. McCrory, A. Parusiński, AlgebraicallyConstructible Functions: Real Algebra and Topology, Real Algebraic and Analytic Geometry RAAG'01, Rennes, June 11-15th, 2001, electronic surveys http://www.maths.univ-rennes1.fr/ raag01/

[15] J.R. Munkres, Elements of algebraic topology, Reading: Addison-Wesley (1984)

[16] D. J. Newman, One-to-one polynomial maps, Proc. A.M.S. 11 (1960), 867-870

[17] A. Parusiński, Z. Szafraniec, Algebraically constructible functions and signs of polynomials, Manuscripta Math. 93 (1997), 443-456. 
[18] A. Parusiński, Z. Szafraniec, On the Euler characteristic of fibres of real polynomial maps, Singularities Symposium - Łojasiewicz 70, Banach Center Publications, PWN, Warszawa, 44 (1998), 175-182

[19] D. Sullivan, Cominatorial invariants of analytic spaces, Proceedings Liverpool Singularities Symposium I, Lecture Notes in Math., vol. 192, Springer, Berlin, 1971, 165-169

Département de Mathématiques, U.M.R. 6093 Du C.N.R.S, Université D'Angers, 2, Bd Lavoisier, 49045 Angers Cedex, France

E-mail address: parus@tonton.univ-angers.fr 